\title{
Research on the Historical Succession and Spatial Distribution Pattern of Rubber Plantation in Xishuangbanna Based on Remote Sensing
}

\author{
Hua Yuan \\ Institute of International Rivers and Eco-Security, Yunnan \\ University, Kunming, Yunnan 650091, PR China \\ Faculty of Forestry, Southwest Forestry University, \\ Kunming, Yunnan 650224, PR China \\ yuanhua_yn@hotmail.com
}

\author{
Cairong Yue ${ }^{\mathrm{b}}$, Wanqiu Zhang ${ }^{*} \mathrm{~d}$ \\ Faculty of Forestry, Southwest Forestry University, \\ Kunming, Yunnan 650224, PR China \\ bcryue@163.com, 'wanqiu_mou@hotmail.com
}

\section{Yali Song}

China Shipbuilding Trading (Kunming) Co. Ltd., Kunming, Yunnan 650051, PR China yalisong@126.com

\begin{abstract}
Based on the phenological regularity of rubber plantation and availability of data, this paper selected the Landsat satellite remote sensing data at 7 phases in America from 1976 to 2010 and combined and object-oriented classification. This means to set threshold based on vegetation index and carry out the decision tree classification first, and then use the decision tree classification results to mask. Then conduct multi-scale segmentation for images in the mask areas with object-oriented method, and carry out feature statistics for the sample plot to extract the information of rubber forest and some young growth with the classification accuracy over 84\%. Combined with DEM data, it analyzed the historical succession and special distribution pattern of rubber plantation according to a time serious from altitude, slope, and aspect. The results show that the planting area of rubber forest has increased by 154 times during the 35 years and rubber planting plots gradually evolve from the low-altitude hills, gentle slope, sunny slope into high-altitude area, abrupt slope and shady areas.
\end{abstract}

Keywords-remote sensing, rubber plantation, historical succession, spatial distribution pattern, Xishuangbanna

\section{INTRODUCTION}

Xishuangbanna is China's second largest rubber plantation area and has the largest area of rain forest and monsoon forest with various plants and creatures. Since 1956, state-run farms have planted rubber, which led to the dramatic increase of population and continuous reduction of natural forest area. Therefore, the ecological environment of this region deteriorated, coupled with ecological contradictions such as water and soil loss, declining soil fertility and local climate change ${ }^{[1-5]}$. Rubber plantation is the dominant type of artificial vegetation in Xishuangbanna. To explore the historical succession and spatial distribution pattern of rubber plantation by remote sensing is of great significance for industry planning and environmental protection in this region. The satellite remote sensing technology could be used to quickly extract rubber plantation acreage and the spatial distribution information, which is conducive to rubber forest monitoring and analysis of dynamic changes, thereby contributing to the local decisions.

\section{AN OVERVIEW OF THE RESEARCH AREA}

Xishuangbanna is located in the southern part of Yunnan province with north latitude $21^{\circ} 10^{\prime}-22^{\circ} 40^{\prime}$ and longitude $99^{\circ} 55^{\prime}-101^{\circ} 50$ ', as shown in Figure 1. It is in the northern edge of the tropic, and belongs to the subtropical monsoon climate, hot and rainy, annual rainfall of 1500-1800mm. It has the alternating wet and dry climate.

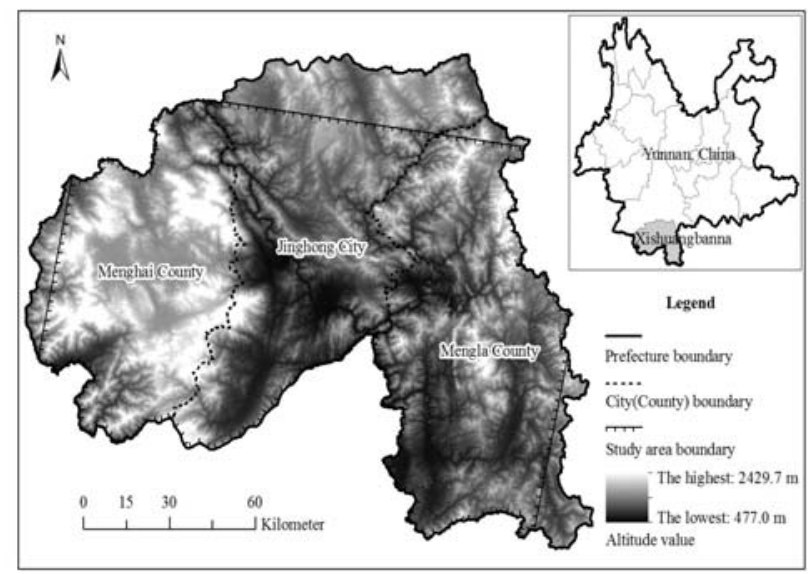

Figure 1. Location of study area

\section{DATA PROCESSING AND RESEARCH METHODS}

\section{A Data sources}

Landsat data has rich archived data, the earliest data from the 1970s, so it is the best choice for the long time-series studies.Data used in this study includes MSS / TM / ETM images at different periods (path/row 130/45, covering more than $80 \%$ of Xishuangbanna, 129 / 45,130 / 44), DEM data and its derived slope, aspect data, field GPS survey data, some high spatial resolution imaging of Google Earth. Taking into account the accessibility of satellite image data and phenological regularity of rubber forest, the MSS / TM / ETM image in this study is mainly from 
January and February and a few of them from June and December, as shown in Table 1.

TABLE 1. REMOTE SENSING DATASETS

\begin{tabular}{c|c}
\hline Acquisition Time & Sensor Type \\
\hline $19750425 / 19760224$ & MSS \\
\hline 19880202 & TM \\
\hline 19921111 & TM \\
\hline 19991225 & ETM \\
\hline 20010112 & ETM \\
\hline 20050216 & TM \\
\hline 20100214 & TM \\
\hline
\end{tabular}

\section{B Data processing}

- $\quad$ Remote sensing data preprocessing

Remote sensing data preprocessing includes radiometric calibration and geometric correction. Radiometric correction adopts histogram adjustment method, while geometric correction adopts polynomial method based on the topographic maps and GPS ground control points to make the accuracy reach 0.5 pixels.

- Interpretation features of rubber plantation

According to the actual ground surveys, most of mature rubber forests are from the state-owned farms, so the area is large and centralized. The images show smooth texture and high coverage rate of vegetation; however, young rubber forests distribution is more dispersed, mainly from private rubber plantation, so the land area is small and texture is rough.

- Rubber plantation information extraction methods

Many scholars have done a lot of researches in these areas to extract rubber plantation information by remote sensing. Zhang Jinghong et al ${ }^{[6]}$ use TM data to establish template for rubber planting area and non-planting area by supervised classification methods. Wang Shudong et al ${ }^{[7]}$ built and optimized the amphibious index WLI, trees and shrubs index GSI, dry land-sand index SSI. Liu Xiaona et al ${ }^{[8]}$ determined the extraction time window of rubber forest by analyzing the phenological characteristics of vegetation cover and adopted the object-oriented classification method according to the spectral differences of rubber forest in different ages. Nian Bo et al ${ }^{[9]}$ utilized the OIF index and combined with vegetation spectral characteristics and various bands of TM5. Li Jun-Ming et al ${ }^{[10]}$ utilized the correlation coefficient matrix, the best band index method and the covariance eigenvalues method to develop the band combination program.

This study conducted a comprehensive analysis of characteristics of the above classification methods and the results indicate that the object-oriented classification method is undoubtedly the best one, but it is difficult to choose image segmentation scale and judge the optimal scale. Therefore, it proposed to adopt decisions tree classification method first, and divide the research area into dense vegetation area and sparse vegetation area based on
NDVI (NDVI means the ratio of the difference between the NIR band and the Red band and their sum), NDBI ${ }^{[11-12]}$ (NDBI means the ratio of the difference between the SWIR band and NIR band and their sum) and DEM. Then mask the dense vegetation area and adopt the easy-to-difficult extraction method for the sparse vegetation area, which means to extract the identifiable un-vegetated area and then conduct small-scale segmentation in order to extract information of rubber young growth with a small area. In the area with dense vegetation, conduct image segmentation and explore the extraction characteristics and rules of rubber forest according to the sample features, then extract the rubber young growth and merge them to form the final distribution map of rubber forests.

\section{$C$ Analysis of the spatial distribution pattern}

Convert the classification of remote sensing images at each phase into raster format, pixel size is set to $30 \mathrm{~m}$ and get the terrain factors such as slope, aspect by the terrain analysis with DEM data. Then conduct spatial overlay analysis for both of them and explore the spatial distribution rules of rubber forest at different periods.

\section{RESULTS AND ANALYSIS}

\section{A Results of remote sensing classification}

Classification accuracy assessment uses ground survey points not involved in classification training samples, and with the aid of Google Earth high-definition images and historical phase images 200 points are selected each period to verify the accuracy, which are up to more than $84 \%$, thus meeting the application requirements. Classification accuracy for each period is respectively $84 \%$ for $1976,87 \%$ for $1988,86 \%$ for $1992,87 \%$ for $1999,93 \%$ for $2001,91 \%$ for 2005 , and $88 \%$ for 2010 . Results of the classification are shown in Figure 3, with the rubber plantation area represented as the white area. As can be seen from the chart, rubber plantations are mainly concentrated in dam area and nearby hilly area of Mengla County, south of Jinghong City, and southwest of Menghai. Besides, Mengla County has the largest area of plantation regardless of in which period, and southern Jinghong and southwest of Menghai have undergone a rapid growth in recent years. 


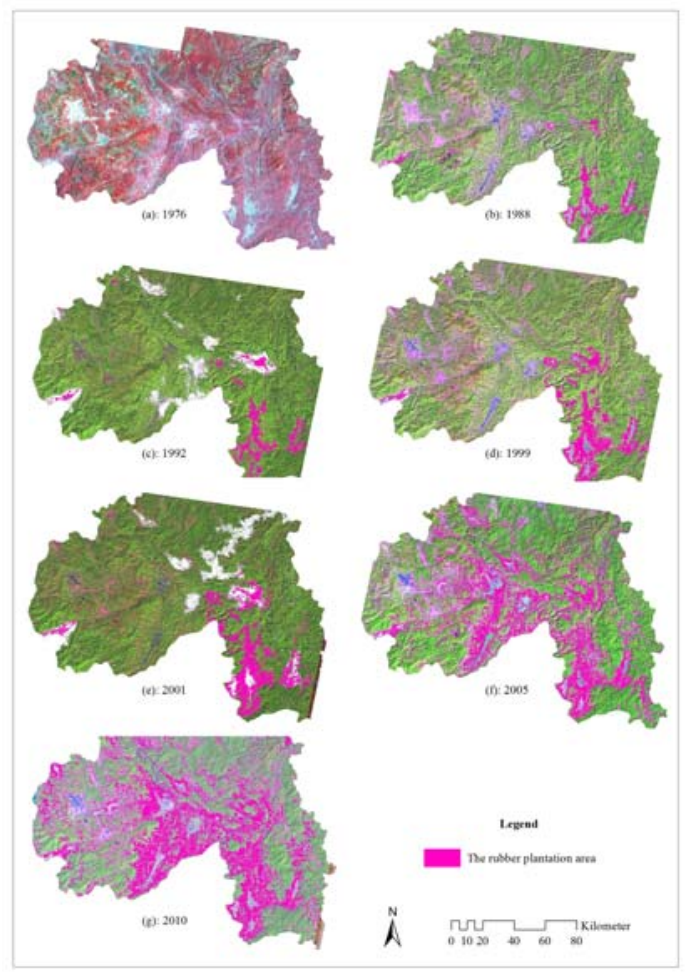

Figure 2. Rubber plantation area of various periods extracted from Landsat datasets

\section{$B$ Analysis of the variation in rubber plantation area}

With the rising price of rubber, a growing trend of private-own rubber has continued since the 1990s, especially in the past decade. The statewide rubber plantation in Xishuangbanna in 1976 was only 2485.3 ha, as opposed to 386,000 ha in 2010, increasing by 154 times. The fastest growing periods are 1976-1988, 1992-1999, 2001-2005, and 2005-2010. The growth trend is consistent with the price trend of dry rubber market ${ }^{[13]}$.

\section{Elevation changes in rubber plantations}

The suitable planting altitude of the rubber trees ranges from $600 \sim 1000 \mathrm{~m}$. Dividing the altitude into $0-600,600-800,800-1000, \quad 1000-1200,1200-1400$, and above $1400 \mathrm{~m}$ and conducting spatial statistics on plaque of rubber plantations in each period, we can obtain a diagram of the correlation between rubber distribution and altitude in each period. It can be seen from Figure 5 that rubber plantations are mainly concentrated in the range of $600-800$ m altitude from 1976 to 2001, while in 2001, they gradually move up to $1000-1200 \mathrm{~m}$, reaching more than $40 \%$ of the total plantations in 2010, and a large scale of cultivation of rubber has initiated in the area above $1400 \mathrm{~m}$. The main reason is that rubber prices increase led to rising enthusiasm of farmers, as there are few low-altitude fields for rubber planting, it is necessary to turn into the high-altitude areas. However, the poor hydrothermal conditions may cause damage to the rubber trees, so the risk exposure is greatly increased.

\section{Rubber plantations slope changes}

The slope is divided into 6 levels, including 0-5 degree, 5-8 degree, 8-15 degree, 15-25 degree, 25-35 degree, above 35 degree, to carry out spatial statistics for each rubber plot and get the average slope. The fields with smaller slope are mainly used as building land and farmland, higher slope is used as woodland. It can be seen from Figure 6 that the rubber was mainly planted in the gentle hills in 8-15 degree in 1976 rubber, followed by the range of 15-35 degree. The dam area with small slope also has some rubber planted, but for the steep slope with the slope above 35 degree, there are no rubber plantations. With time goes by, there are no available fields with small slope for rubber plantation, so areas with the slope ranging from 15-25 degree and 25-35 degree have been developed, but the areas with the slope above 35 degree are not due to the inconvenient traffic.

\section{E Changes of rubber plantations aspects}

To simplify the statistics, divide aspects into three categories: ground (no aspect), shady slope and sunny slope, as shown in Figure 7. Ground and sunny slope are exposed to the sunshine, but the shady aspect fails to be exposed to the sunshine. Rubber growth requires heat, so sunny slope, especially the southern aspect, is more suitable for cultivation. From 1976 to 2010, southern aspect is the most important cultivation area, but the proportion shows annual decrease and the distribution of rubber in shady slope is increasing, and this indicates that the sunny slope is in short. Generally, ground is located in the flatland and the peak area, for the flatland is mainly used as urban land and agricultural land and rarely used for rubber planting. Planting rubber at shady slope also increases the risk of frost damage.

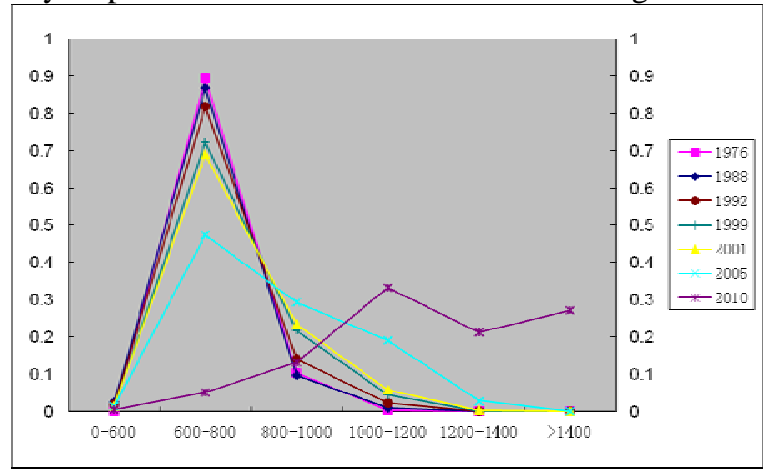

Figure 3. Relationship between rubber plantation areas and different altitude 


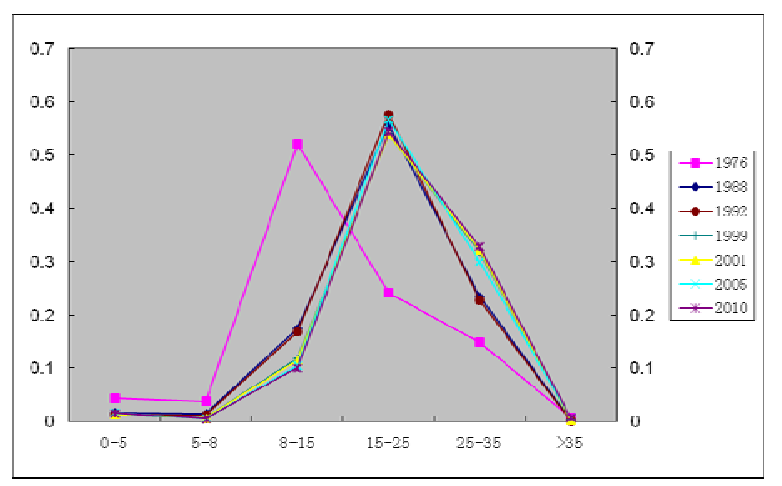

Figure 4. Relationship between rubber plantation areas and different slopes

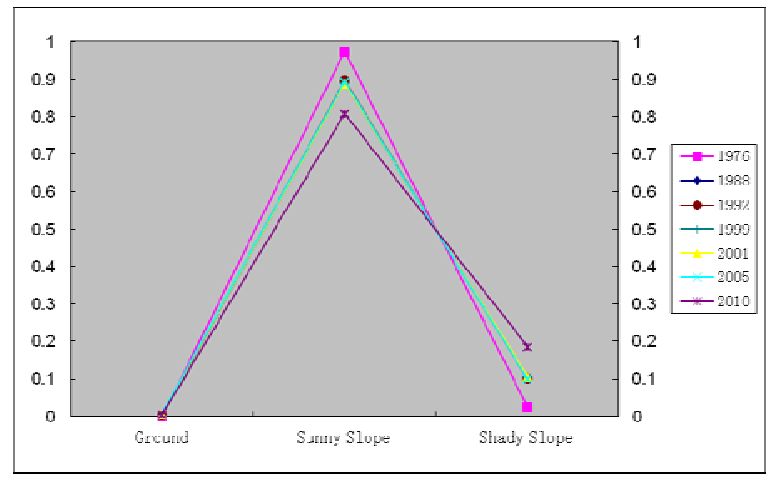

Figure 5. Relationship between rubber plantation areas and 3 aspects

\section{$\mathrm{V}$ CONCLUSION AND DISCUSSION}

Based on the phenological regularity of rubber plantation and availability of data, this paper selected the Landsat satellite remote sensing datasets of 7 phases from 1976 to 2010 and combined and object-oriented classification. This means to set threshold based on vegetation index and carry out the decision tree classification first, and then use the decision tree classification results to mask it. Then conduct multi-scale segmentation for images in the mask areas with object-oriented method, and carry out feature statistics for the sample plot to extract the information of rubber forest and some young growth with the classification accuracy over $84 \%$. Combined with DEM data, it analyzed the historical succession and special distribution pattern of rubber plantation according to a time serious from altitude, slope, and aspect.

With the rise of rubber prices, private rubber plantation area also exceeded state farms and rubber planting plots gradually evolve from the low-altitude hills, gentle slope, sunny slope into high-altitude, abrupt slope, shady areas. While new species of rubber have been improved in production and cold resistance, the poor heat conditions of high-altitude area and shady area still impact the yield of rubber and increase the risk of chilling damage. Unlimited expansion of rubber plantation area will bring supply and demand saturation, resulting in profits reduction of rubber planting. Using the rubber to replace tropical monsoon forest may influence the water conservation, soil and water preservation and even the local climate change to a certain degree, so it is necessary to explore the relationship between local ecological environment change and rubber plantations.

\section{ACKNOWLEDGMENT}

This work was financially supported by National Key Discipline of Forest Management support from Southwest Forestry University (XKZ200901). We thank the anonymous reviewers for their suggestions to improve this manuscript.

\section{CORRESPONDING AUTHOR}

Zhang Wanqiu. Tel.: +86 87163863022 E-mail address: wanqiu_mou@hotmail.com

\section{REFERENCES}

[1] Zhou Zong, Hu Shaoyun. Research on the Influence of Rubber Industry on Ecological Environmental of Xishuangbanna [J] Environmental Science Survey, 2008 (3): 73-75.

[2] Zhou Zong, Hu Shaoyun. Tan Yingzhong. Rubber Plantations in large area in Xishuangbanna and Its Impact on Ecological Impact [J] Yunnan Environmental Science, 2006 (S1): 67-69.

[3] Zhou Wai, Wu Zhaolu, He Qincheng. Rubber Plantations and Water Shortages: Case Study of GeNiu Village in Xishuangbanna [J] Journal of Ecology, 2011 (7): 1570-1574.

[4] Yang Weimin, Qin Wei. The Impact of the Development of Rubber in Xishuangbanna of Yunnan Province on Ecological Environment [J] Ecological and Economics (Academic Edition), 2009 (1): 336-339.

[5] Zhang Peifang, Xu Jianchu, Wang Maoxin, et al. Rubber Plantation Characteristics in Xishuangbanna and Its Influence on the Tropical Forest Landscape Characteristics based on Remote Sensing [J] Land and Resources, 2006 (3): 51-55.

[6] Zhang Jinghong, Tao Zhongliang, Liu Shaojun, etc. Information Extraction of Rubber Planting Area in Hainan based on TM Images [J] Chinese Journal of Tropical Crops, 2010 (4): 661-665.

[7] Wang Shudong, Zhang Lifu, Chen Xiaoping, et al. Extraction Model and Method of Trophic Fine Surface Features based on Landsat TM --- An Cased Study in Hainan Island [J] Journal of Ecology, 2012 (22): 7036-7044.

[8] Liu Xiaona, Feng Zhiming, Jiang Luguang, et al. Remote Sensing and Digital Mapping of Xishuangbanna Rubber Forest [J] Resources Science, 2012 (9): 1769-1780.

[9] Nian Bo, Yang Shijian, Wang Jinliang. The Best Band Selection of Extracted Vegetation Remote Sensing Information - A Case Study in the Central Mountainous Area of Yunling [J] Yunnan Geographic Environment Research, 2004 (2): 18-21.

[10] Li Junming, Xing Yanqiu, Yang Chao. Research on the Optimal Band Selection of Spectral Characteristics of Forest Types - Take HJ / 1A Hyperspectral Imaging as Example [J] Forest Engineering, 2013 (4): 42-46.

[11] Yang Zhixiang, He Xiufeng. Automatic Extraction of Urban Land Information of Remote Sensing Images based on the Improved NDBI Index [J] Journal of Hohai University (Natural Science), 2010 (2): 181-184.

[12] Yang Yunyuan, Shi Zhengtao, Xu Chengdong, et al. Automatic Extraction of Urban Information based on Landsat TM [J] Sensing Technology and Application, 2009 (5): 654-659 + 554.

[13] Qi Dongling, Wang Xiuquan, Zhang Zhiyang, et al. China's Natural Rubber Industry Status Quo and Development Proposals [J] Tropical Agricultural Sciences, 2013 (2): 79-87. 